\title{
Corrigendum: A Novel Microtubule-Tau Association Enhancer and Neuroprotective Drug Candidate: Ac-SKIP
}

\author{
Yanina Ivashko-Pachima and Illana Gozes* \\ Dr. Diana and Zelman Elton (Elbaum) Laboratory for Molecular Neuroendocrinology, Department of Human Molecular \\ Genetics and Biochemistry, Sackler Faculty of Medicine, Sagol School of Neuroscience, Adams Super Center for Brain \\ Studies, Tel Aviv University, Tel Aviv, Israel
}

Keywords: tau, microtubules, EBs, ADNP, SKIP

\section{A Corrigendum on}

\section{OPEN ACCESS}

Edited and reviewed by:

Dirk M. Hermann

University of

Duisburg-Essen, Germany

${ }^{*}$ Correspondence: Illana Gozes

igozes@tauex.tau.ac.il; igozes@post.tau.ac.il

Specialty section:

This article was submitted to

Cellular Neuropathology,

a section of the journal

Frontiers in Cellular Neuroscience

Received: 29 March 2021

Accepted: 28 April 2021

Published: 26 May 2021

Citation:

Ivashko-Pachima Y and Gozes I

(2021) Corrigendum: A Novel Microtubule-Tau Association Enhancer and Neuroprotective Drug Candidate:

Front. Cell. Neurosci. 15:687301. doi: 10.3389/fncel.2021.687301

\section{A Novel Microtubule-Tau Association Enhancer and Neuroprotective Drug Candidate: Ac-SKIP \\ by Ivashko-Pachima, Y., and Gozes, I. (2019). Front. Cell. Neurosci. 13:435. doi: $10.3389 /$ fncel.2019.00435}

In the original article, there were inconsistencies when comparing one by one the panels of Figure $4 \mathrm{~A}$ in the main text and the representative extended immunoblot results in Supplementary Figure S3, as published. Thus, some of the representative images in the main text were unfortunately represented by extended mirrored, duplicated and rotated blots as explained below. It should be emphasized that for scientific rigor and data corroboration, each experiment was performed in triplicates and repeated three times and each immunoblot was exposed at least three times (three films), hence, the blot shown as a full image in the original supplementary material is a representative picture, as specifically stated in the original Supplementary Figure S3 legend. To maintain scientific rigor, there were 36 blots and almost 100 films. Our mistakes, corrected below, stem from an unfortunate mix-up coupled with an extremely high similarity between all repetitive experiments, we apologize for the mistakes.

Corrections have been made to Figure 4, panel A, and Supplementary Figure S3. The corrected figures are shown below.

The authors apologize for these errors and state that they do not change the scientific conclusions of the article in any way. The original article has been updated.

Copyright $\odot 2021$ Ivashko-Pachima and Gozes. This is an open-access article distributed under the terms of the Creative Commons Attribution License (CC BY). The use, distribution or reproduction in other forums is permitted, provided the original author(s) and the copyright owner(s) are credited and that the original publication in this journal is cited, in accordance with accepted academic practice. No use, distribution or reproduction is permitted which does not comply with these terms. 
A

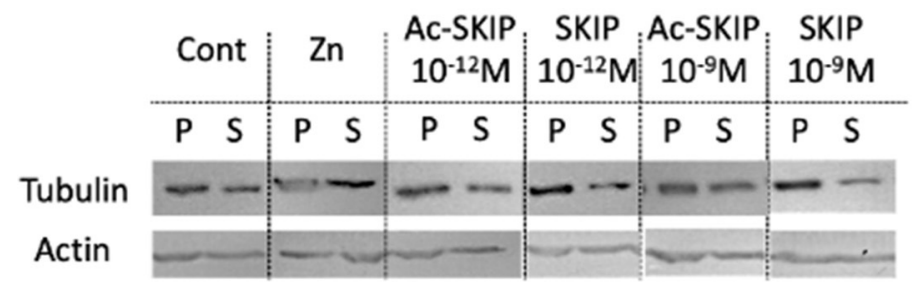

C

IP: Neg IP: EB1 IP: EB1 IP: EB1

Cont Cont SKIP AC-SKIP

E1 E2 E1 E2 E1 E2 E1 E2

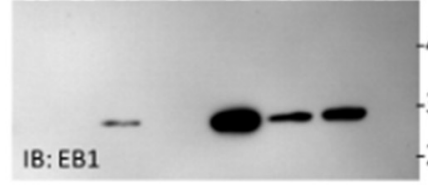

IP: EB1 IB: EB1

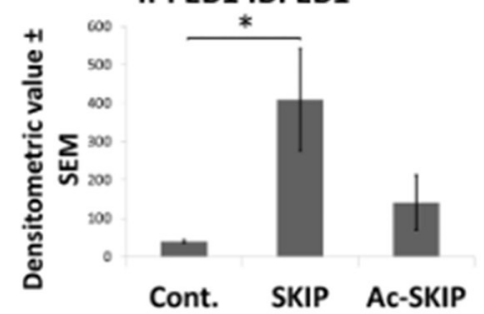

D

IP: Neg IP: Tau IP: Tau IP: Tau Cont Cont SKIP AC-SKIP

E1 E2 E1 E2 E1 E2 E1 E2
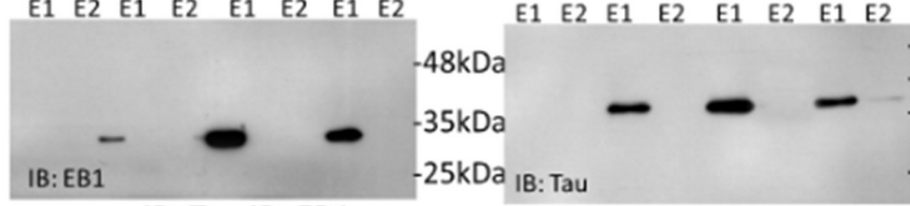

IP: Tau IB: EB1

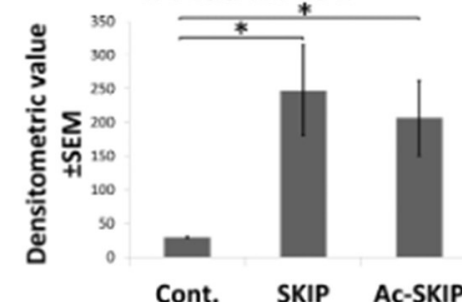

E $\begin{array}{llll}\text { IP: Neg IP: EB1 IP: EB1 IP: EB1 } & \end{array}$

Cont Cont SKIP AC-SKIP

E1 E2 E1 E2 E1 E2 E1 E2
B

$25 k D a$ IB: Tau
IP: EB1 IB: Tau

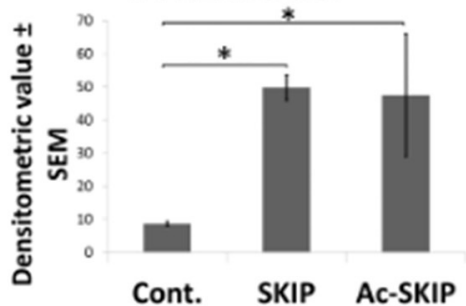

Cont. SKIP Ac-SKIP
IP: Neg IP: Tau IP: Tau IP: Tau Cont Cont SKIP AC-SKIP
IP: Tau IB: Tau

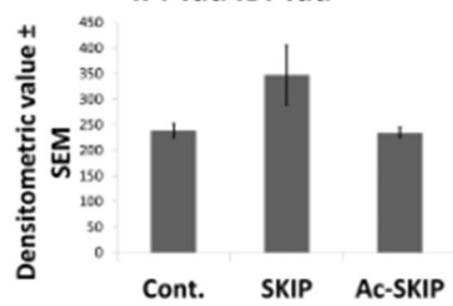
Input EB1 Tau Tubulin

Tubulin

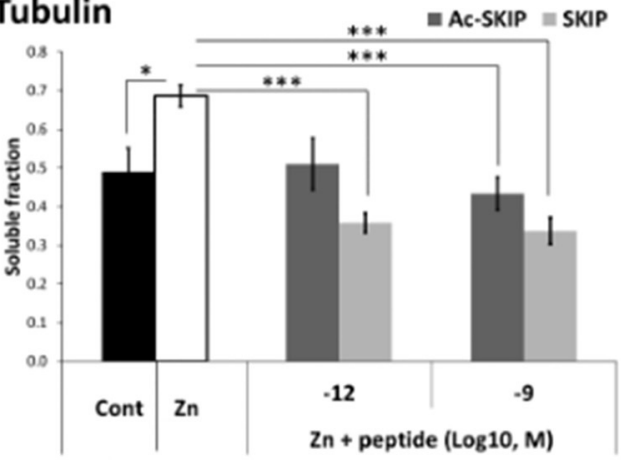

IP: Neg IP: EB1 IP: EB1 IP:EB1

Cont Cont SKIP AC-SKIP

E1 E2 E1 E2 E1 E2 E1 E2

$-75 \mathrm{kDa}$

$-63 k D a$

$75 \mathrm{kDa}$

$48 \mathrm{kDa}$

$-35 k D a$ IB: Tubulin

$63 \mathrm{kDa}$

$48 \mathrm{kDa}$

IP: EB1 IB: Tubulin

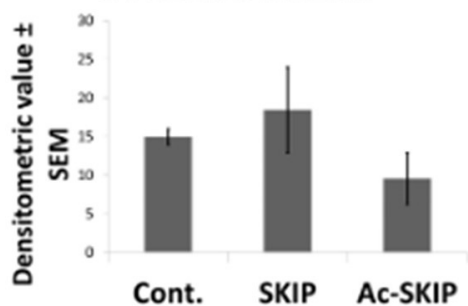
$35 \mathrm{kDa}$

IP: Neg IP: Tau IP: Tau IP: Tau Cont Cont SKIP AC-SKIP E1 E2 E1 E2 E1 E2 E1 E2 $75 \mathrm{kDa}$ $-63 k D a$

$-48 \mathrm{kDa}$

-35kDa IB: Tubulin

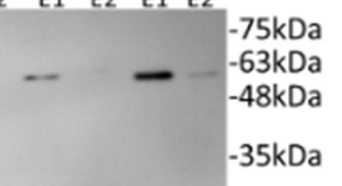

IP: Tau IB: Tubulin

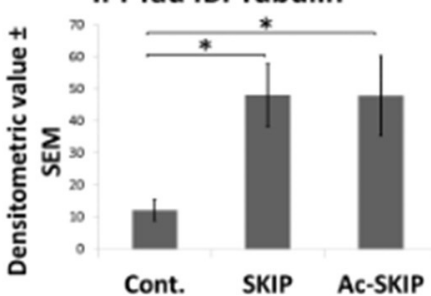

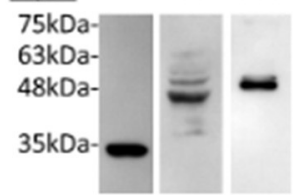

FIGURE 4 | Ac-SKIP and SKIP effect on the polymerized vs. the soluble tubulin pool and the crosstalk between EB-Tau-tubulin. (A) Immunoblotting of polymerized (P) and soluble (S) protein fractions (obtained by polymerized vs. soluble tubulin assay, see section "Materials and Methods") with tubulin antibodies. Cells were treated with zinc $(400 \mu \mathrm{M}, 4 \mathrm{~h})$ with or without Ac-SKIP or SKIP $\left(10^{-12} \mathrm{M}\right.$ and $\left.10^{-9} \mathrm{M}, 4 \mathrm{~h}\right)$, non-treated cells served as controls. (B) The graph represents the densitometric 
FIGURE 4 | quantification of soluble tubulin ratios. The intensity of each band was quantified by densitometry and the soluble protein ratio was calculated by dividing the densitometric value of soluble proteins by the total protein content (S/[S + P]). Statistical analysis was performed by One Way ANOVA with Tukey HSD. ${ }^{*} P<0.05$, ${ }^{* *} \mathrm{P}<0.01,{ }^{* *+*} \mathrm{P}<0.001$; Control, $n=15 ; \mathrm{Zn}, n=18 ; \mathrm{Zn}+$ Ac-SKIP $10^{-12} \mathrm{M}, n=15 ; \mathrm{Zn}+$ SKIP $10^{-12} \mathrm{M}, n=9 ; \mathrm{Zn}+\mathrm{Ac}-\mathrm{SKIP} 10^{-9} \mathrm{M}, n=18 ; \mathrm{Zn}+\mathrm{SKIP} 10^{-9} \mathrm{M}$, $n=11$. (C,D) A Co-IP assay was performed with EB1 or Tau antibodies, linked to agarose beads. SKIP ( $2 \mu \mathrm{g} / \mathrm{sample})$ or Ac-SKIP (2 $\mu \mathrm{g} / \mathrm{sample})$, diluted into Pierce lysis buffer (see section "Materials and Methods") or the equal volume of lysis buffer w/o peptides (IP: EB1 Cont; IP: Tau Cont) were added to cell lysate of differentiated SH-SY5Y cells, 15 min before EB1 or Tau column application (IP: EB1; IP: Tau). Sequential IP elution fractions (E1, E2) were further analyzed by immunoblotting with EB1, Tau, and Tubulin antibodies (IB: EB1; IB: Tau; IB: Tubulin). In addition, columns with free agarose beads were used as negative controls (IP: Neg cont). The intensity of each band was quantified by densitometry. The bar graph shows the ratio of band intensities (densitometric value \pm SEM) obtained upon SKIP and Ac-SKIP treatments as compared with non-treated cells (Cont). Statistical analysis was performed by One Way ANOVA with LSD HSD. Experiments were independently repeated three times. ${ }^{*} P<0.05,{ }^{* *} P<0.01,{ }^{* * *} P<0.001$. (E) Cell lysates without column exposure were used as positive controls (Input). IP immunoprecipitation, IB - immunoblot. 
A

\section{Tubulin}

Cont

123

$P S P S P S$

50

$\mathrm{kDa}$
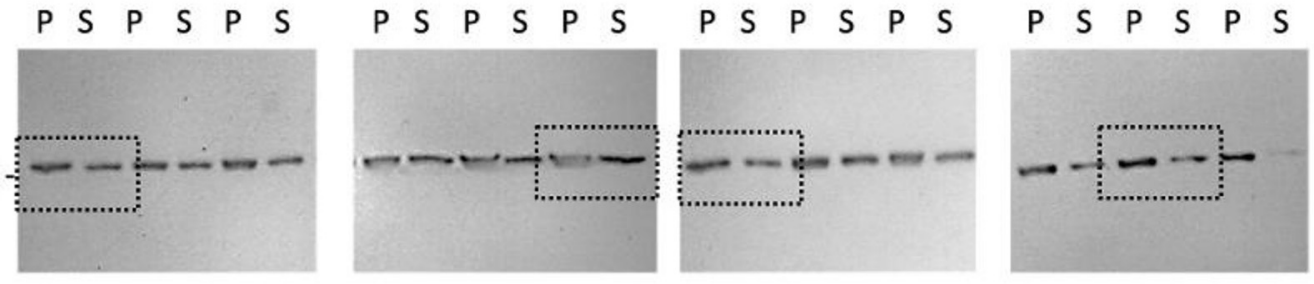

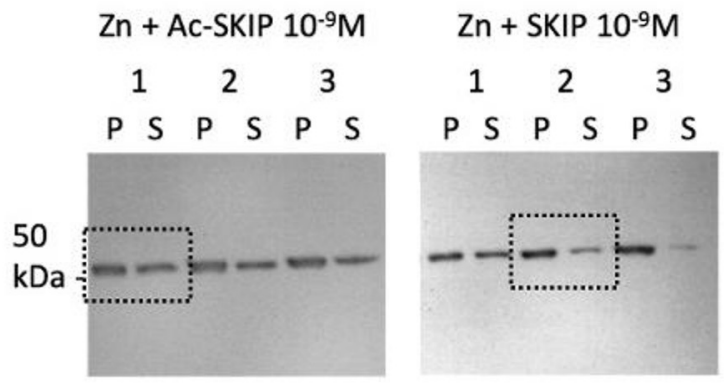

B
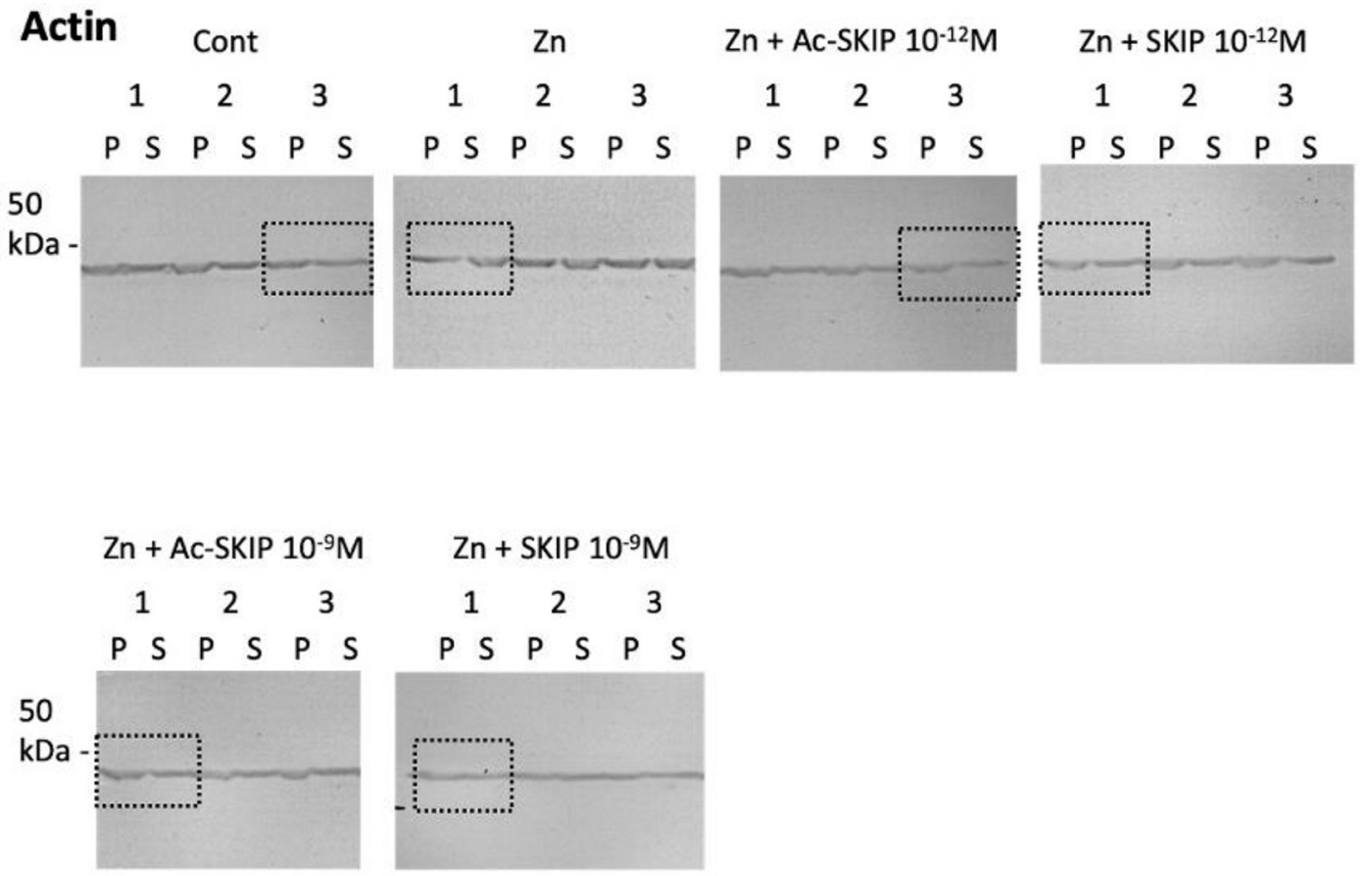

SUPPLEMENTARY FIGURE S3 | Representative pictures of immunoblot done with tubulin (A) and actin (B) antibodies. Polymerized (P) and soluble (S) tubulin pools were obtained from lysed differentiated N1E-115 cells without treatment (Control) or treated with zinc alone or together with $\mathrm{Ac}-\mathrm{SKIP}$ or SKIP at $10^{-12} \mathrm{M}$ and $10^{-9} \mathrm{M}$. Dotted squares represent areas, cut and displayed in the Figure 4 of the main text. 